\title{
Étude sur la transversalité des pratiques critiques d'adolescents québécois dans le cadre de leurs cours de sciences, d'histoire et d'éthique
}

\section{Mathieu Gagnon}

Cet article présente les résultats d'une étude exploratoire sur la transversalité des pratiques critiques d'élèves du secondaire. Cette étude prend appui, d'une part, sur l'analyse d'activités d'apprentissage par problèmes menées en classe de sciences, d'histoire et d'éthique à partir d'une grille structurée autour d'interventions constitutives des pratiques critiques et, d'autre part, sur des entretiens menés auprès d'élèves et d'enseignants. Entre autres résultats, l'auteur indique que les pratiques critiques sont des pratiques situées et que leur transversalité s'inscrit à l'intérieur d'un processus complexe et dynamique pouvant être examiné à partir de quatre pôles, à savoir la discipline, les espaces de résolution, le type de problème ainsi que les rapports aux savoirs.

Penser la transversalité à travers une dialogique entre le générique et le spécifique

En éducation, la transversalité est généralement examinée à l'aune des notions de compétence (Rey, 1998, 2011) et/ou de capacité, et ce, principalement à l'intérieur des différents plans d'études (Plan d'études romand, PER), programmes de formation (Programme de formation de l'école québécoise, PFEQ) ou décrets (Décret définissant les missions prioritaires de l'enseignement fondamental et de l'enseignement secondaire et organisant les structures propres à les atteindre, Belgique). Seulement, l'idée même de compétence transversale soulève nombre de questions et est remise en doute depuis plusieurs années (Gagnon, 2008a; Lafortune et al., 2011; Rey, 1998). Différentes raisons participent à cette ambivalence à l'égard des compétences transversales, parmi lesquelles se retrouvent notamment le manque réel de recherches empiriques à leur sujet ainsi que la mise en évidence d'une contradiction apparente entre la transversalité, associée à une perspective "générique», et l'idée que toute compétence est située. Cette tension entre les caractères générique et spécifique des compétences et/ ou capacités transversales s'exprime d'ailleurs à l'intérieur des différents plans d'études ou programmes de formation. À cet égard, notons que le PER (Suisse) 
inscrit davantage sa définition à l'intérieur d'une perspective située, alors que le PFEQ propose une approche plus proprement générique. En effet, à l'intérieur du PER, il est dit que les capacités transversales «sont mobilisées au travers de nombreuses situations contextualisées leur permettant de se développer et d'étendre progressivement leur champ d'application", tandis qu'à l'intérieur du PFEQ, le ministère de l'Éducation, du Loisir et du Sport du Québec indique que ces compétences sont dites transversales «en raison de leur caractère générique» (MELS, 2006, p. 11).

Seulement, à la lumière de nos analyses autour de la notion de compétence transversale (Gagnon, 2008a), il nous est apparu que la transversalité, tout en désignant "ce qui traverse une grande variété de situations», ne pouvait être entièrement séparée du contexte dans lequel les actions prennent forme. En ce sens, nous avons posé que la transversalité ne peut ni être réduite à des familles de situations, ni être pensée de manière totalement générique et décontextualisée, ni s'appuyer sur une logique de transfert, jugée aporétique, voire contre productive. Ainsi, notre position s'inscrit davantage dans une perspective de cognition située (Brown, Collins \& Duguid, 1989; Perkins \& Salomon, 1989) et de complexité (Morin, 1986) qui s'enracine, selon les mots de Perrenoud (2002), non pas par le truchement de la métaphore du transfert, mais bien de celle de la mobilisation, entendue au sens d'une mobilisation transversale. De sorte que notre posture nous conduit à adopter une épistémologie dialogique dont l'une des visées, dans le cas de la transversalité, consiste à penser ensemble le spécifique et le générique, l'émergeant et le transcendant, le disciplinaire et le transdisciplinaire, etc. Dit autrement, qu'il soit possible d'exercer une compétence dite transversale à l'intérieur d'une grande variété de situations, lesquelles sont parfois fort différentes les unes des autres par les notions qu'elles convoquent ou encore les démarches qu'elles sollicitent, est une chose, mais par là nous ne sommes pas justifiés de soutenir que cette compétence, lorsque maîtrisée dans un cadre spécifique ( $\mathrm{p}$. ex. un contexte disciplinaire donné) sera maîtrisée dans tous les contextes par la même personne. De fait, pour s'exercer, une compétence transversale doit s'appuyer, notamment, sur une série de connaissances, d'habiletés et d'attitudes, dont plusieurs demeurent spécifiques aux domaines. De sorte que bien que bon nombre de composantes associées à une compétence transversale puissent être exprimées de manière générique ou transdisciplinaire, leurs mobilisations et combinaisons ne peuvent faire fi de la spécificité des situations dans lesquelles elles prennent forme.

\section{La transversalité de la pensée critique: quelles questions pour la recherche en éducation?}

À l'intérieur des différents documents officiels cités précédemment, les conduites associées à la pensée, à l'esprit, au sens ou au jugement critique sont rangées 
sous la catégorie des compétences ou capacités dites transversales. Or, qu'elle soit considérée comme une compétence, une capacité, une disposition, une conduite ou encore comme une pratique, l'hypothèse de la transversalité de la pensée critique, quoique discutée dans les écrits et supposée pour une large part dans les différentes définitions, soulève nombre de questions qui appellent la mise en route d'études plus proprement empiriques. Entre autres questions, notons: comment penser ensemble le caractère spécifique de chacune des situations dans lesquelles la pensée critique est mobilisée et la perspective générique habituellement associée à la transversalité? Comment joindre les idées d'actions situées et de transversalité, alors que cette dernière renvoie d'ordinaire à une perspective transdisciplinaire (Jonnaert, 2002)? La pensée critique peut-elle être considérée comme une "compétence» suffisamment générale pour qu'elle puisse être mobilisée à l'intérieur de toute situation, peu importe le contexte (Rey, 1998, 2011)? Doit-on voir la transversalité, ainsi que le développement transversal de la pensée critique, de manière contextualisée ou décontextualisée? La transversalité de la pensée critique doit-elle être liée à des familles de situations (Fourez, 2005) ou, de par sa nature même, est-elle appelée à les dépasser? Que la pensée critique puisse être mobilisée dans des contextes variés par différentes personnes implique-t-il qu'une seule personne puisse être transversalement critique? Autant de questions, et bien d'autres, qui, pour l'heure, ne retrouvent pas de réponses claires dans les écrits et sur lesquelles le présent article souhaite diriger l'attention.

Depuis ses origines, la pensée critique a souvent été considérée comme étant transdisciplinaire, et son caractère dit générique s'exprime notamment par le biais des composantes auxquelles elle est associée, parmi lesquelles nous retrouvons, entre autres, les termes thinking skills, dispositions, abilities, higher-order thinking skills, capacities, etc. (Bailin, 2002; Barell, 1991; Brookfield, 1987; Ennis, 1987, 1993; Facione \& Facione, 1996; Halpern, 1989; Kurfiss, 1988; Landis, Swain, Friehe \& Coufal, 2007; Lipman, 2003; Norris \& Ennis, 1989; Paul, 1990; Siegel, 1985). Cependant, la question de la transversalité de la pensée critique s'est cristallisée à travers les échanges menés entre McPeck et Ennis, lesquels marquent clairement les tensions entre son supposé caractère générique et transdisciplinaire, et la spécificité des situations dans lesquelles elle prend forme. Pour McPeck (1981, 1990), les manières dont se construisent les pratiques critiques sont si dépendantes de la particularité des différents domaines (compte tenu, notamment, de la spécificité des corpus qui les composent), qu'il est difficile de concevoir qu'elles puissent être vues comme génériques et «transférables». Ennis quant à lui (1989), comme d'autres (Brell, 1990; Lipman, 2003; Norris, 1992; Paul, 1990; Siegel, 1992; Smith, 2002), est plutôt d'avis que les particularités liées aux manières dont se met en route la pensée critique à l'intérieur des différents domaines n'en contredit pas la généricité, et ce, dans la mesure où chacun partagerait des structures profondes communes qu'il suffit d'adapter, en quelque sorte, aux particularités des circonstances dans lesquelles elles prennent forme. 
Dans ce cas, l'adaptation présumée représente davantage une différence de degré que de nature. Cependant, malgré sa prise de position, Ennis (1989) conclut que cette question nécessite la mise en route de recherches empiriques afin de documenter plus avant cet enjeu, notamment en ce qui a trait aux manifestations d'une pensée critique à l'intérieur de différents domaines (dont les matières scolaires). Bien que posé en 1989, cet appel à des recherches empiriques ne retrouve, encore aujourd'hui, que fort peu d'écho dans la littérature (Billing, 2007). De fait, la plupart des écrits s'intéressant au caractère transversal de la pensée critique soit demeurent essentiellement d'ordre théorique (Brell, 1990; Smith, 2002), soit s'inscrivent à l'intérieur d'un seul domaine par le recours au concept de transfert et ne visent pas à engager des analyses comparatives entre les domaines ou matières scolaires. Dès lors, sous l'angle de la recherche, la question de l'«existence» d'une pensée critique comme capacité ou compétence transversale demeure entière.

C'est à ce besoin qu'entend contribuer la présente étude, laquelle était guidée par la question suivante: De quelle manière des élèves de quatrième secondaire (14-15 ans) mobilisent-ils leur pensée critique, s'ils le font, à l'intérieur de matières scolaires différentes? En ce sens, notre principal objectif de recherche consistait à mettre au jour des indices d'une possible transversalité de la pensée critique, et ce, dans diverses matières scolaires au secondaire. Mais avant d'exposer les paramètres méthodologiques et les résultats obtenus lors de cette étude, prenons soin de clarifier la conception de la pensée critique sur laquelle se sont appuyés nos travaux.

\section{Quels cadres pour l'étude de la transversalité de la pensée critique d'adolescents?}

Que la transversalité de la pensée critique soit un enjeu de fond pour l'éducation ne repose pas uniquement sur les questions que soulève à elle seule l'idée de transversalité, mais bien sur la conception largement partagée selon laquelle la pensée critique représente une "compétence» nécessaire à la vie courante en tant qu'elle est liée à l'autonomie de pensée et d'agir dans un monde hautement complexe, un monde dans lequel chacun sera appelé à exercer sa citoyenneté afin de faire raisonnablement face aux défis, nombreux et cruciaux, du $21^{\mathrm{e}}$ siècle (De Koninck, 2007; Delors, 1996; Jonas, 1999; UNESCO, 2008). Or, au-delà de cette volonté éducative partagée en occident quant à la formation de la pensée critique, demeure l'importance des cadres à partir desquels seront mis en route non seulement les interventions éducatives en favorisant le développement, mais également la structuration des paramètres de recherche en permettant l'analyse. À cet égard, s'appuyant sur une démarche réflexive visant à analyser différents processus de conceptualisation de la pensée critique depuis les travaux de Dewey (1933), nous en sommes venus à proposer une définition qui prend en 
compte son mode propre, à savoir le mode évaluatif, et qui permet de penser à la fois le générique et le spécifique tout en pouvant s'inscrire à l'intérieur de notre conception de la transversalité, laquelle est fondée sur les principes de cognition située et de mobilisation tels que décrits précédemment (Gagnon, 2011b). Ainsi, nous définissons la pensée critique comme une pratiquel évaluative fondée sur une démarche réflexive, autocritique et autocorrectrice impliquant le recours à différentes ressources (connaissances, habiletés de pensée, attitudes, personnes, informations, matériel) dans le but de déterminer ce qu'il y a raisonnablement lieu de croire (au sens épistémologique) ou de faire (aux sens méthodologique et éthique) en considérant attentivement les critères de choix et les diversités contextuelles (Gagnon, 2010, 2011a, 2011d). Cette définition indique que la pensée critique est envisagée sous l'angle d'une pratique située qui gagne à être appréciée en contexte (Brookfield, 1987), avec tout ce que cela comprend d'attitudes (et/ou d'émotions), d'habiletés (et/ou de cognition), de diversité et de complexité. Elle tend également à éviter les pièges de l'organisation des ressources, notamment cognitives, selon des critères de complexité interne ou de linéarité (Gagnon \& Sasseville, 2009)2 laissant ainsi ouvertes les perspectives de la complexité contextuelle, de l'itérativité de même que de la (co)combinaison en situation. Dans ce cas, le sens téléologique des pratiques critiques (i.e. déterminer ce qu'il y a lieu de croire ou de faire) sert de guide à la (co)combinaison des ressources, alors que le contexte, quant à lui, sert de cadre à la complexité et à l'itérativité des processus.

C'est en prenant appui sur cette définition que nous avons entrepris notre étude. Celle-ci ne visait pas en premier lieu à déterminer le niveau de pensée critique des élèves, ni à examiner dans quelle mesure ils procédaient à son transfert. Nous souhaitions, selon les indications de Ennis (1989), examiner leurs pratiques critiques lorsqu'ils sont invités à s'engager dans des processus de résolutions de problèmes dits complexes ${ }^{3}$ associés à des paradigmes disciplinaires variés. Les parties qui suivent seront consacrées à la présentation ainsi qu’à l'analyse des résultats obtenus lors de cette étude. Mais d'abord, précisons les éléments contextuels et méthodologiques de la recherche.

\section{Principaux éléments contextuels et méthodologiques de la recherche}

Les présents résultats sont issus d'une étude visant à comparer les pratiques critiques manifestées par quinze élèves (divisés en trois sous-groupes) de quatrième secondaire à l'intérieur de cinq activités d'apprentissage par problèmes $(\mathrm{APP})^{4}$ menées en classe de sciences ( 2 activités de 75 minutes), d'éthique (1 activité de 300 minutes) et d'histoire ( 2 activités de 75 minutes) ${ }^{5}$. Le choix des élèves s'est effectué suivant un processus d'uéchantillonnage» théorique et de convenance (Lessard-Hébert, Goyette \& Boutin, 1990). Théorique, parce qu'ils ont été sélectionnés en raison de leur quatre années scolaires d'expérience avec la 
communauté de recherche philosophique (CRP) et que, selon certaines études, cette approche permet d'engager les élèves à l'intérieur de pratiques critiques et contribue au développement de leur pensée critique (Daniel \& Gagnon, 2011). Échantillonnage de convenance également, puisqu'ils ont été choisis en fonction de la possibilité de les observer à l'intérieur d'un même sous-groupe dans l'ensemble des trois matières scolaires retenues. À ce sujet, notons que nous avons retenu l'éthique puisque les élèves ont exercé leur pensée critique à l'intérieur des CRP dans lesquelles les questions éthiques sont au cœur de la démarche. Quant aux cours de sciences et d'histoire, ceux-ci ont été retenus, notamment, parce qu'ils constituent des cours obligatoires et qu'ils appartiennent à des domaines différents, à savoir les sciences humaines et les sciences de la nature, permettant ainsi d'effectuer des expérimentations à l'intérieur de situations n'appartenant pas nécessairement aux mêmes «familles».

Cette recherche visait principalement la formulation d'hypothèses (Maren, 2003) et comprenait deux volets: descriptif et comparatif. Le volet descriptif servait à dessiner un portrait contextualisé des pratiques critiques observées à l'intérieur de chacune des activités, alors que le volet comparatif visait à identifier des tendances générales se dégageant des activités menées, d'abord à l'intérieur d'une seule matière scolaire (analyses comparatives intradisciplinaires), puis dans les différentes matières scolaires (analyses comparatives interdisciplinaires). L'observation participante a servi de mode de collecte de données lors des activités et le chercheur questionnait les élèves de manière non systématiquement planifiée (De Ketele \& Roegiers, 1993).

L'analyse des activités menées en classe a été effectuée à l'aide d'une grille construite autour d'interventions constitutives des pratiques critiques (Gagnon, 2011b). Les types d'interventions utilisés sont les suivants: interventions non-justifiée; justifiée; critériée; éthique; contextuelle; évaluative; épistémique; métacognitive; autocritique et autocorrectrice ${ }^{6}$. Chacune de ces interventions peut porter soit sur un «objet» (manifestation d'un point de vue), sur ses propres pratiques (comme individu ou comme groupe), sur les propos des pairs, sur ceux des experts, sur l'information ou sur le matériel ${ }^{7}$. Aucune d'entre elles n'est cependant suffisante en elle-même ni mutuellement exclusive, et chacune des tendances relevées a été examinée dans son contexte d'apparition par une analyse du discours in situ.

Des entrevues semi-dirigées (Stewart \& Cash, 1982) d'une durée de 75 minutes ont également été menées auprès des cinq sous-groupes d'élèves observés et des enseignants. Celles-ci portaient principalement sur la place accordée à la réflexion critique dans chacune des matières scolaires à l'étude et visaient à compléter les données ainsi qu'à vérifier la cohérence interne des résultats. Les informations obtenues par le biais de ces entretiens ont été traitées par le recours à une analyse de contenu dans laquelle les différentes unités de sens ont été classifiées à l'aide d'une grille à catégories mixtes (L'Écuyer, 1990). Trois axes principaux ont été identifiés: 1) ce qu'est la pensée critique; 2) ses champs d'application; et 3) sa place dans les différentes matières scolaires (Gagnon, 2009). 
Examinons maintenant ce qui ressort des données eu égard à la transversalité des pratiques critiques des élèves observés.

\section{Principaux résultats de recherche: analyses comparatives intra et interdisciplinaires}

Nous débuterons la présentation des résultats en nous appuyant sur ce que nous pourrions qualifier de doxa de la transversalité, à savoir selon une approche par analyse comparative intra et interdisciplinaire. Le tableau 1 présente les occurrences relevées quant aux différentes interventions en fonction des activités et des matières scolaires. Soulignons que ces données, de nature quantitative, servent essentiellement de guides ou de repères à l'analyse qualitative du discours en marquant des tendances.

Compte tenu des contraintes inhérentes au présent texte, nous dirigerons l'attention sur certains des aspects qui, à la lumière des données présentées, nous sont apparus déterminants dans la compréhension que nous pourrions avoir de la transversalité des pratiques critiques. Soulignons d'abord que le tableau 1 montre au moins trois tendances que nous pourrions qualifier de transversales dans les pratiques (voire l'absence de pratique) des élèves, à savoir 1) la majorité des interventions demeurent non-justifiées; 2) dans l'ensemble, très peu d'interventions constitutives des pratiques critiques portent sur les experts, l'information ou le matériel; 3) les interventions éthiques, autocritiques, autocorrectrices et épistémiques sont celles qui ont été le moins mobilisées par les élèves dans la plupart des activités.

Partant, malgré qu'il soit possible de relever une certaine transversalité dans les pratiques des élèves, il n'est pas pour autant possible, sur la base de ces données, d'inférer une transversalité certaine, et ce, pour différentes raisons. L'une d'entre elles nous provient des données associées aux déclinaisons des interventions, lesquelles marquent parfois des écarts entre les disciplines, voire entre deux activités menées à l'intérieur d'une même discipline. À titre d'exemples, notons que bien que la majorité des interventions codées soient considérées non-justifiées, cette absence de justification (du moins sous l'angle de l'observable) portait en sciences ainsi qu'en histoire sur l'information (activités sur le tableau périodique et sur la $2^{\mathrm{e}}$ guerre mondiale) et le matériel (activité sur le concept de mole). En ce sens, un regard sur les interventions évaluatives relevées montre que pour ces mêmes activités, très peu d'entre elles portent sur l'information ou le matériel lors des activités en sciences, alors qu'il s'agit de la déclinaison la plus importante lors de l'activité sur la $2^{\mathrm{e}}$ guerre. Semblablement, alors que les interventions critériées en sciences portent majoritairement sur l'information ou le matériel, l'analyse ne relève pas une tendance similaire quant à l'activité sur la 2 e guerre. Par ailleurs, nous relevons que l'activité sur le patrimoine comprend, en proportion, un très fort pourcentage d'interventions non-justifiées qui, en comparaison, est plus élevé que celui de la seconde activité en histoire, lequel se compare 


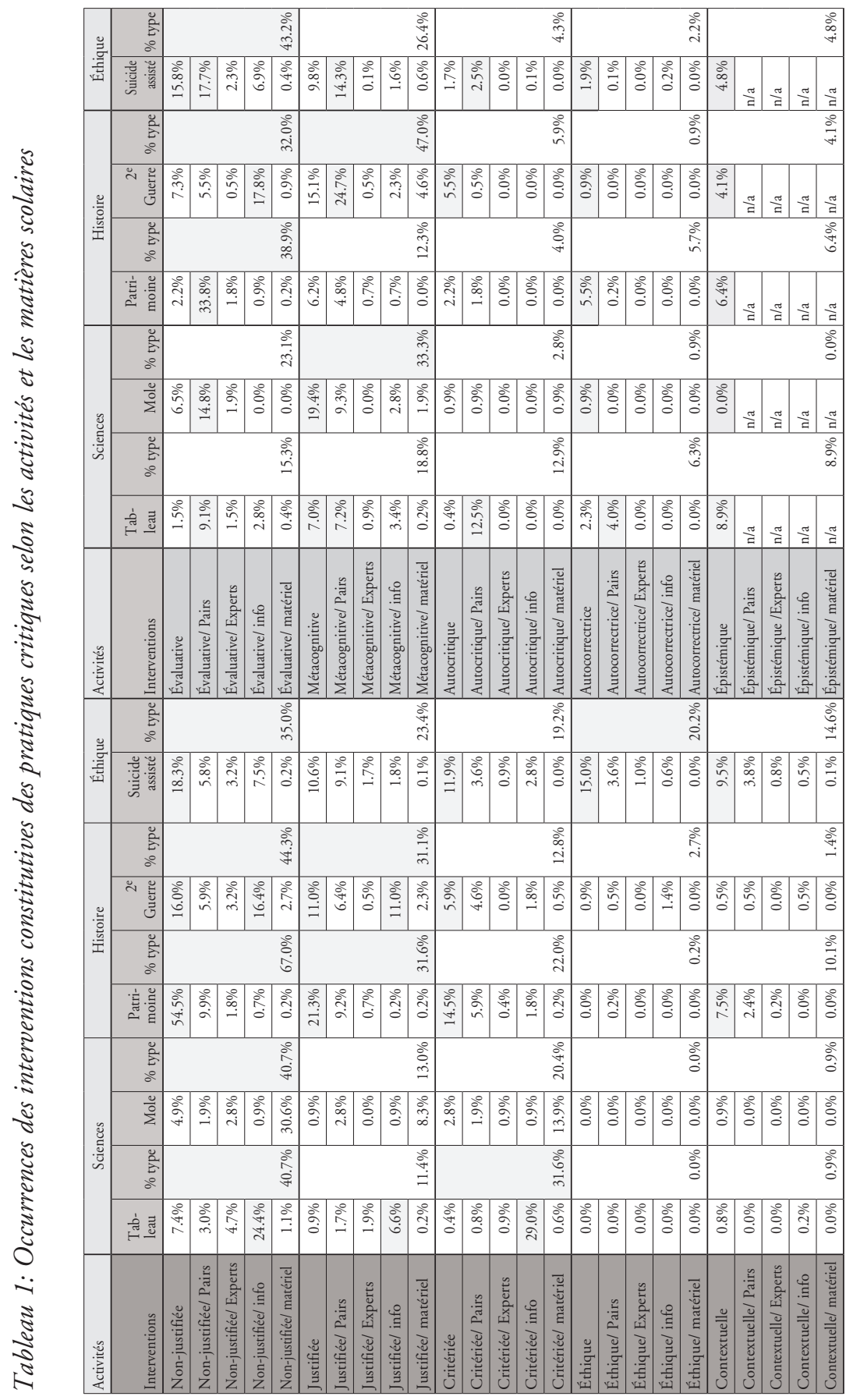


davantage au taux identifié transversalement. Nous relevons également que le taux des interventions contextuelles relevées lors de l'activité sur le patrimoine se compare davantage à celui de l'activité sur le suicide assisté (éthique) qu'à celui de l'activité sur la $2^{\mathrm{e}}$ guerre (histoire), qui lui se compare aux autres occurrences relevées. De même, les interventions autocorrectrices ont été mobilisées plus régulièrement dans les activités sur le tableau périodique et sur le patrimoine (deux domaines différents) qu'à l'intérieur de toutes autres activités. Cependant, alors qu'en sciences (tableau périodique) les autocorrections portaient sur les pratiques du groupe, celles en histoire (patrimoine) se rapportaient à des conduites individuelles.

Dès lors, comment expliquer ces variations, et avec elles la question de la transversalité, si entre deux activités menées à l'intérieur d'une même matière scolaire, nous relevons des écarts parfois plus grands que ceux présents entre les matières scolaires? La réponse à une telle question appelle, selon nous, un retour aux processus de résolution. À cet égard, nous alimenterons notre analyse in situ par le recours à trois tableaux qui reprennent le relevé des interventions, mais cette fois en comparant les activités sur la base des espaces de résolution (discussion initiale, classification et/ou recherche d'information; plénière) ${ }^{9}$. Ainsi, nous pourrons examiner si un regard porté sur le type de problème permet de rendre compte d'une forme de transversalité ${ }^{10}$ (voir tableau 2).

Tableau 2: Occurrences des interventions constitutives des pratiques selon les espaces de résolution - Discussion initiale

\begin{tabular}{|c|c|c|c|c|c|c|c|c|c|}
\hline \multicolumn{10}{|c|}{ Espaces de résolution } \\
\hline & \multicolumn{2}{|c|}{ Patrimoine } & \multicolumn{2}{|c|}{ Suicide assisté } & & \multicolumn{2}{|c|}{ Patrimoine } & \multicolumn{2}{|c|}{ Suicide assisté } \\
\hline Non-justifiée & $8.9 \%$ & \multirow{5}{*}{$\begin{array}{l}13,9 \% \\
(1,9 \%) \\
{[3,6 \%]}\end{array}$} & $17.6 \%$ & \multirow{5}{*}{$\begin{array}{c}27,8 \% \\
(6,6 \%) \\
{[18,8 \%]}\end{array}$} & Évaluative & $6.3 \%$ & \multirow{5}{*}{$\begin{array}{c}27,8 \% \\
(3,8 \%) \\
{[12,4 \%]}\end{array}$} & $20.8 \%$ & \multirow{5}{*}{$\begin{array}{c}46,1 \% \\
(11 \%) \\
{[25,4 \%]}\end{array}$} \\
\hline Non-justifiée/ Pairs & $0.0 \%$ & & $2.0 \%$ & & Évaluative/ Pairs & $17.7 \%$ & & $19.2 \%$ & \\
\hline $\begin{array}{l}\text { Non-justifiée/ } \\
\text { Experts }\end{array}$ & $5.1 \%$ & & $4.9 \%$ & & Évaluative/ Experts & $3.8 \%$ & & $4.5 \%$ & \\
\hline Non-justifiée/ info & $0.0 \%$ & & $3.3 \%$ & & Évaluative/ info & $0.0 \%$ & & $1.6 \%$ & \\
\hline $\begin{array}{l}\text { Non-justifiéel } \\
\text { matériel }\end{array}$ & $0.0 \%$ & & $0.0 \%$ & & Évaluative/ matériel & $0.0 \%$ & & $0.0 \%$ & \\
\hline Justifiée & $22.8 \%$ & & $18.4 \%$ & & Métacognitive & $15.2 \%$ & & $10.2 \%$ & \\
\hline Justifiée/ Pairs & $10.1 \%$ & & $10.6 \%$ & & Métacognitive/ Pairs & $5.1 \%$ & & $2.4 \%$ & \\
\hline Justifiée/ Experts & $2.5 \%$ & & $4.5 \%$ & & Métacognitive/ Experts & $0.0 \%$ & & $0.0 \%$ & \\
\hline Justifiée/ info & $0.0 \%$ & & $0.8 \%$ & $34,7 \%$ & Métacognitive/ info & $0.0 \%$ & $\begin{array}{l}20,5 \% \\
(28 \%)\end{array}$ & $0.4 \%$ & $\begin{array}{l}15,1 \% \\
(3,1 \%)\end{array}$ \\
\hline Justifiée/ matériel & $0.0 \%$ & $11.4 \%$ & $0.4 \%$ & {$[35,3 \%]$} & Métacognitive/ matériel & $0.0 \%$ & {$[28,6 \%]$} & $0.0 \%$ & {$[11,8 \%]$} \\
\hline Critériée & $27.8 \%$ & & $16.7 \%$ & & Autocritique & $0.0 \%$ & & $3.3 \%$ & \\
\hline Critériée/ Pairs & $6.3 \%$ & & $3.7 \%$ & & Autocritique/ Pairs & $1.3 \%$ & & $0.4 \%$ & \\
\hline Critériée/ Experts & $1.3 \%$ & & $1.6 \%$ & & Autocritique/ Experts & $0.0 \%$ & & $0.0 \%$ & \\
\hline Critériée/ info & $0.0 \%$ & $\begin{array}{l}35,4 \% \\
(4.8 \%)\end{array}$ & $0.8 \%$ & $\begin{array}{l}22,9 \% \\
(5,4 \%)\end{array}$ & Autocritique/ info & $0.0 \%$ & $\begin{array}{l}1,5 \% \\
(0,2 \%)\end{array}$ & $0.0 \%$ & $(0,9 \%)$ \\
\hline Critériée/ matériel & $0.0 \%$ & {$[26,9 \%]$} & $0.0 \%$ & {$[28,3 \%]$} & Autocritique/ matériel & $0.0 \%$ & {$[0,8 \%]$} & $0.0 \%$ & {$[20,5 \%]$} \\
\hline Éthique & $0.0 \%$ & & $28.6 \%$ & & Autocorrectrice & $0.0 \%$ & & $3.3 \%$ & \\
\hline Éthique/ Pairs & $0.0 \%$ & & $4.9 \%$ & & Autocorrectrice/ Pairs & $0.0 \%$ & & $0.0 \%$ & \\
\hline Éthique/ Experts & $0.0 \%$ & & $3.3 \%$ & & Autocorrectrice/ Experts & $0.0 \%$ & & $0.0 \%$ & \\
\hline Éthique/ info & $0.0 \%$ & & $0.0 \%$ & & Autocorrectrice/ info & $0.0 \%$ & & $0.0 \%$ & \\
\hline Éthique/ matériel & $0.0 \%$ & $0.0 \%$ & $0.0 \%$ & $\begin{array}{l}(8,7 \%) \\
{[43,3 \%]}\end{array}$ & $\begin{array}{l}\text { Autocorrectrice/ } \\
\text { matériel }\end{array}$ & $0.0 \%$ & $0.0 \%$ & $0.0 \%$ & $\begin{array}{c}(0,8 \%) \\
{[34,8 \%]}\end{array}$ \\
\hline
\end{tabular}




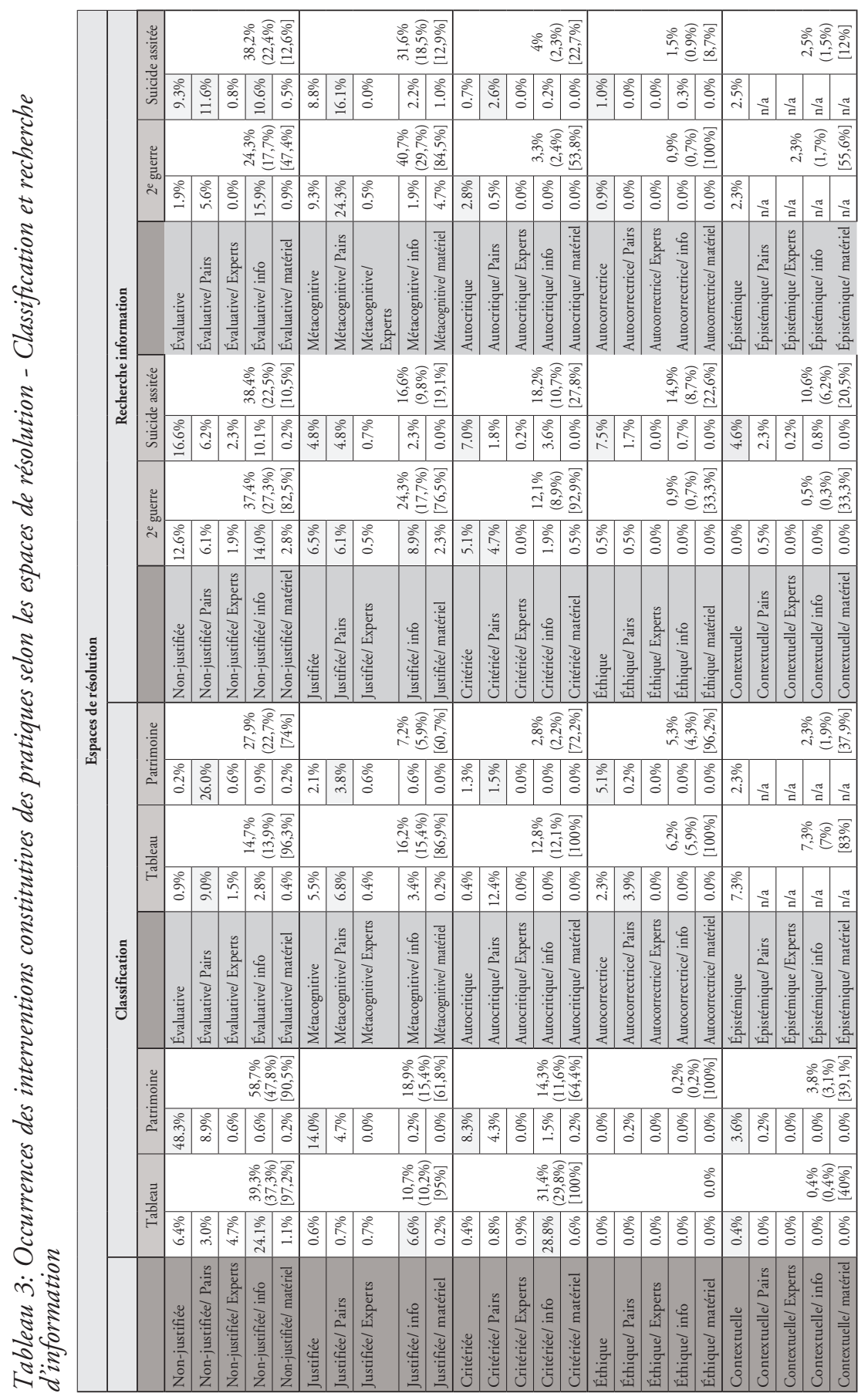




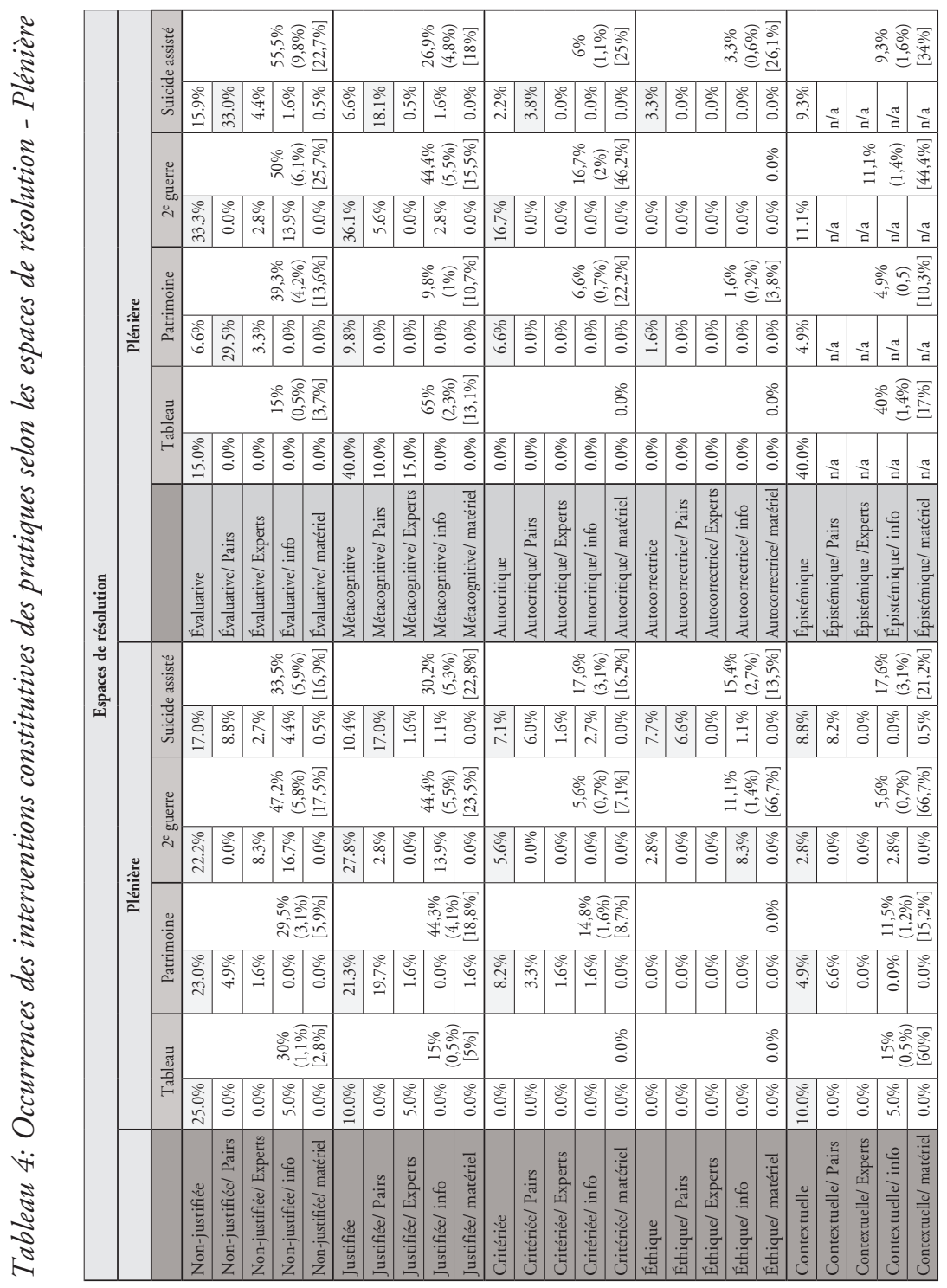


Ces trois tableaux nous montrent qu'il est possible d'observer une forme de transversalité en prenant appui sur les espaces de résolution, lesquels représentent en quelque sorte des problèmes d'un certain type. De fait, selon cet axe, plusieurs activités présentent des pourcentages d'occurrences somme toute très près les uns des autres selon les interventions, et ce, malgré que dans la plupart des cas les espaces comparés sont associés à des disciplines différentes. De plus, ces tableaux semblent faire voir certaines «tendances lourdes» en fonction des espaces. À titre d'exemple, notons que dans le cadre des activités sur le tableau périodique ainsi que sur le patrimoine, c'est près de $90 \%(88,8 \%)$ de toutes les interventions critériées, autocritiques et autocorrectrices mobilisées qui se retrouvent à l'intérieur de l'espace classification. Semblablement, dans certains espaces, une forte propension de toutes les interventions constitutives mobilisées se rapporte à un certain type. C'est le cas par exemple des interventions métacognitives qui, lors de la plénière de l'activité sur le tableau périodique, comptent pour $65 \%$ des interventions codées dans cet espace alors que ce type ne représente en fait que $2,3 \%$ de toutes les interventions codées pour cette activité. Une tendance similaire est observable concernant les activités sur la $2^{\mathrm{e}}$ guerre ainsi que le suicide assisté. En outre, notons que pour un même type d'interventions, il est possible de relever des écarts entre les espaces, selon le type de problème à résoudre. Cet aspect est très bien illustré par les interventions autocorrectrices, qui sont davantage présentes lors des activités de classification (tableau périodique et patrimoine) qu'elles ne le sont dans le cadre des activités de recherche d'information ( $2^{\mathrm{e}}$ guerre et suicide assisté).

Cependant, à l'image de ce qu'il nous a été possible de relever lors des analyses comparatives intra et interdisciplinaires inscrites au tableau 1, la transversalité se dégageant de l'axe «espaces de résolution» n'est ni absolument intégrale, ni absolument systématique. En effet, alors que 15\% des interventions codées lors de la plénière sur le tableau périodique sont de nature évaluative, elles représentent près de $50 \%$ des interventions pour les autres activités. De même, alors que $93 \%$ des interventions critériées mobilisées lors de l'activité sur la $2^{\mathrm{e}}$ guerre l'ont été lors de la recherche d'information, elles ne représentent que $28 \%$ de celles qui ont été mobilisées lors de l'activité sur le suicide assisté pour le même espace. Néanmoins, selon l'angle à partir duquel nous abordons les données (voir note 10), ces écarts entre les activités selon les espaces de résolutions sont moins importants que ceux relevés entre les activités d'une même discipline ou encore entre les disciplines (tableau 1).

Les données issues de cette analyse font voir qu'une approche quantitative décontextualisée, pour peu qu'elle nous fournit des repères importants, ne peut suffire. Ainsi, afin de penser la complexité de ces liens et de faire émerger des hypothèses explicatives quant à ces tendances qui, tantôt marquent une forme de transversalité, tantôt nous conduisent à la nuancer, il nous apparaît fondamental de restituer ces données, par le biais d'une approche qualitative, dans leur contexte d'émergence. 


\section{Discussion: penser la transversalité en contexte}

L'analyse des processus in situ, alimentée par le relevé des interventions mobilisées ainsi que des données issues des entretiens, ont permis de noter qu'au moins trois facteurs qui, sans être des déterminants absolus, semblent intervenir dans la transversalité des pratiques critiques des élèves, à savoir 1) la discipline (matière scolaire); 2) les espaces de résolution; 3) le type de problème à résoudre. À ces trois éléments, que nous qualifions de pôles de la transversalité, s'en ajoute un quatrième, relevé lors des entretiens menés auprès des élèves et des enseignants ainsi que lors de certaines activités, à savoir les rapports aux savoirs (Gagnon, 2009). L'identification de ces quatre pôles nous a conduit à construire un modèle systémique de la mobilisation transversale de la pensée critique comme pratique située, illustré de manière iconique par la figure 1.

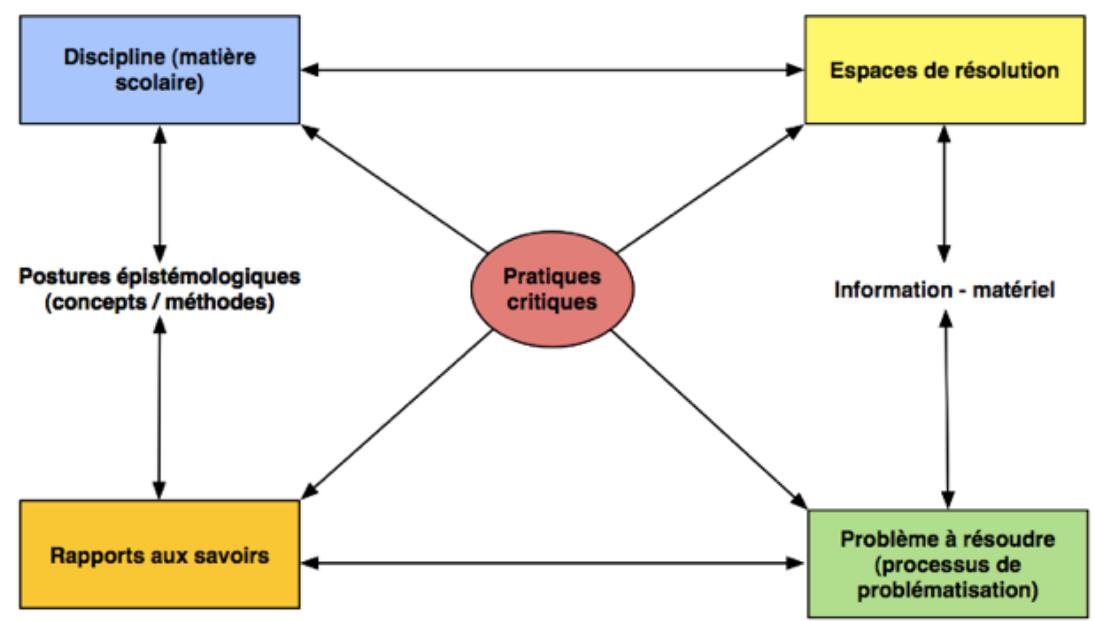

Figure 1: Modèle systémique de la mobilisation transversale de la pensée critique comme pratique située

Ces quatre pôles, lorsque considérés de manière in situ, contribuent à expliquer à la fois la transversalité ainsi que les écarts relevés entre les disciplines, les activités et les espaces de résolution. Prenons soin d'illustrer cette interprétation par quelques exemples. D'abord, la concentration des interventions éthiques à l'intérieur de l'activité sur le suicide assisté témoigne de l'importance de la discipline dans laquelle s'inscrivent les pratiques. Seulement, la discipline n'est pas le seul facteur, comme en témoigne la présence de ce type d'intervention à l'intérieur de l'activité sur la $2^{\mathrm{e}}$ guerre, une activité qui lors de la plénière comprenait un moment où les élèves devaient indiquer dans quelle mesure l'implication des 
femmes avait eu des impacts positifs ou négatifs sur elles. Ainsi, la nature de la question posée est venue en quelque sorte déterminer le type d'interventions mobilisées. Il en va de même pour les interventions épistémiques relevées lors de la plénière sur le tableau périodique, une plénière dans laquelle les élèves étaient appelés à réfléchir sur les processus d'élaboration des modèles scientifiques, leur adéquation à la «réalité» ainsi que leur valeur de vérité. En ce sens, et cet aspect touche le pôle des rapports aux savoirs sous l'angle du contrat didactique, les élèves diront que si le problème posé par l'enseignant et les questions qu'il leur adresse ne vont pas dans le sens de tel ou tel type d'intervention, ils ne la mobiliseront pas, même s'ils y songent (Gagnon, 2009).

Poursuivons sous l'angle des rapports aux savoirs en indiquant que pour les élèves et les enseignants, certains concepts ou questions sont plus propices à l'exercice d'une pensée critique que d'autres. Ainsi en est-il, par exemple, des questions éthiques qui, considérées plus «subjectives» ou "relativistes», sont vues comme favorisant davantage la mise en ouvre de pratiques critiques que les questions plus proprement scientifiques ou historiques, lesquelles participent davantage (toujours selon les élèves) d'une posture à tendance réaliste et positiviste. Semblablement, plus un concept sera considéré stable ou "chauve», moins les élèves ont tendance à s'engager dans des pratiques critiques alors que, inversement, plus un concept est considéré polysémique ou "chevelu», plus ils mobiliseront d'interventions constitutives des pratiques critiques (Gagnon, 2011a, 2011d). Cette question des concepts nous reconduit à celle des disciplines ainsi que des processus de résolution qui leur sont associés. Elle permet également d'expliquer en partie certains des écarts entre les activités. À ce sujet, indiquons que puisque les élèves considéraient le concept de patrimoine comme étant évolutif et culturel alors qu'ils adoptaient un posture plus objectiviste eu égard aux éléments du tableau périodique, le processus de résolution dans chacune de ces activités, bien que «semblable» sous l'angle de la forme, a conduit à des pratiques critiques différentes. Cela explique, par exemple, que davantage d'interventions non-justifiées ont été relevées à l'intérieur de l'activité sur le patrimoine alors que celles notées pour le tableau périodique portaient essentiellement sur l'information; c'est pourquoi également davantage d'interventions critérées, métacognitives et autocritiques ont été relevées lors de l'activité sur le tableau périodique, le problème conduisant à organiser l'information selon des critères définis dans les paramètres de l'activité et devant conduire à une forme de classification et d'organisation communes, tandis que les critères associés au patrimoine devaient être déterminés et pouvaient varier en fonction des contextes, des époques, voire des personnes... C'est pourquoi d'ailleurs les processus d'autocorrection observés se rapportent aux démarches du groupe en sciences (tableau périodique), alors qu'elles étaient liées à des conceptions individuelles en histoire (patrimoine).

Cet aspect nous semble lié au type de problème à résoudre, qui est lui-même lié à la discipline dans laquelle il prend forme. De sorte que pour deux processus 
de classification, malgré les points de convergence notés entre les matières, il demeure des aspects distincts. À cet égard, notons d'abord qu'en sciences (tableau périodique), la démarche s'est avérée plus algorithmique et centrée sur l'information disponible en regard des caractéristiques des éléments, alors qu'en histoire (patrimoine), le processus des élèves était davantage itératif et centré sur l'évaluation du poids des critères en fonction des contextes. Cela explique, notamment, pourquoi l'activité sur le patrimoine a donné lieu à davantage d'interventions contextuelles ainsi que d'interventions non-justifiées, lorsque les élèves étaient tous d'accord pour considérer un item comme faisant partie du patrimoine - en ce sens, notons que lorsqu'il y avait désaccord, les interventions étaient clairement plus justifiées et critériées. Semblablement, la recherche ainsi que l'évaluation de l'information ne se présentaient pas tout à fait sous la même forme selon que l'activité avait lieu en histoire ( $2^{\mathrm{e}}$ guerre) ou en éthique. En effet, alors que l'évaluation de l'information en histoire visait principalement à déterminer si un texte se rapportait au thème à documenter (elle ne comprenait donc aucun processus de cognition épisémique), cette évaluation, en éthique, portait davantage sur les propos émis dans les écrits et prenait en compte les circonstances particulières associées à chacun des cas, expliquant ainsi la plus grande présence d'interventions contextuelles. Cette variation se manifeste d'ailleurs à l'intérieur d'une même activité. De fait, la recherche d'information menée par l'équipe devant documenter le volet économique du suicide assisté s'apparentait davantage à celle observée lors de l'activité sur la $2^{\mathrm{e}}$ guerre qu'à celle des équipes chargées de documenter le volet éthique du suicide assisté. Cette ressemblance s'explique notamment par les rapports aux savoirs partagés par les élèves qui considèrent les données économiques et historiques comme étant davantage objectives que celles relevant plus spécifiquement de l'éthique (Gagnon, 2011d). Cela indique que ces rapports sont davantage liés à la discipline qu'à la matière scolaire à proprement parler. C'est pourquoi d'ailleurs, à la lumière de ces résultats, nous parlons désormais d'espaces de conceptions épistémologiques (Gagnon, 2011a).

\section{Conclusion}

La complexité à laquelle nous faisons face lors de l'analyse transversale des pratiques critiques impliquerait, bien entendu, des développements plus étayés, plus fins et plus nuancés. Dans les faits, à l'image de ce que nous avons présenté lors d'autres publications complémentaires à celle-ci, le poids de l'analyse qualitative est beaucoup plus grand qu'il n'y paraît dans la discussion. Néanmoins, cette étude exploratoire aura conduit à élaborer une conception de la transversalité, une définition des pratiques critiques, une grille d'analyse fondée sur les interventions constitutives des pratiques critiques, une démarche méthodologique ainsi qu'un modèle systémique. Seulement, ces élaborations demeurent 
somme toute peu éprouvées, posant ainsi l'importance de mener des études plus ambitieuses afin de tester nos hypothèses et nos processus d'analyse. Parmi ces hypothèses, nous examinons actuellement les relations possibles entre les pratiques critiques et les rapports aux savoirs des élèves, vues sous l'angle de la transversalité.

Néanmoins, les résultats obtenus lors de cette étude exploratoire nous ont permis d'identifier, en matière de pédagogie et de didactique, des aspects qu'il convient de prendre en compte lorsque nous souhaitons ouvrer au développement de la pensée critique des élèves, a fortiori à son développement transversal. Parmi ces aspects, certains nous semblent particulièrement importants. L'un d'entre eux touche les rapports aux savoirs, lesquels peuvent se décliner en au moins cinq types: 1) rapport épistémologique; 2) rapport épistémique; 3) rapport didactique; 4) rapport idiosyncrasique; 5) rapport gnoséologique (pour une présentation détaillée des éléments ayant conduit à l'élaboration de cette typologie ainsi que pour une définition de ces différents rapports, le lecteur peut consulter le texte de Gagnon, 2011c). Parmi ceux-ci, les rapports épistémologiques, épistémiques et didactiques semblent particulièrement déterminants dans les manières dont les élèves s'engageront à l'intérieur de pratiques critiques. De fait, d'autres études que nous avons menées (Gagnon, 2011a) tendent à montrer que les pratiques critiques des élèves varient beaucoup en fonction des conceptions qu'ils ont de la nature des savoirs dans les différentes disciplines. Ainsi, de manière générale, les élèves se montrent plus critiques en éthique, un peu moins en histoire et très peu en sciences. Cela nous semble attribuable, en partie, à la conception qu'ils ont de la valeur de vérité des savoirs (rapport épistémique) associés à ces différents domaines et des démarches qui permettent de les élaborer (rapport épistémologique). De sorte que, plus les élèves pensent que les savoirs sont obtenus par la découverte de faits objectifs, moins ils auront tendance à mettre en œuvre des pratiques évaluatives. Le rapport didactique quant à lui est lié, en contexte scolaire, à ces rapports épistémique et épistémologique. En effet, plus un domaine est considéré comme étant structuré autour de faits non questionnables, plus les enseignants et les élèves auront tendance à s'inscrire dans une dynamique pédagogique selon laquelle le rôle principal du maître consiste à «transmettre» des informations que les élèves doivent mémoriser, ce qui, du coup, contribue à diminuer les espaces permettant l'exercice d'une pensée critique.

En somme, il ressort de cette étude que si nous souhaitons favoriser, chez les élèves, une mobilisation de plus en plus transversale de leur pensée critique, le simple fait de la développer à l'intérieur d'une matière scolaire spécifique ne saurait suffire, compte tenu, notamment, de la variation des rapports aux savoirs qui est en jeu. Du coup, cela signifie que tous les enseignants doivent offrir aux élèves des espaces pour qu'ils puissent s'exercer à penser de manière critique. Partant, tant l'organisation de leurs situations d'apprentissage que leurs interventions en classe gagneraient à prendre en compte les différentes dimensions associées à l'exercice d'une pensée critique. Semblablement, il semble qu'une 
formation épistémologique pour les enseignants de toutes les disciplines contribuerait largement à favoriser ce développement transversal de la pensée critique, car les croyances épistémologiques des enseignants semblent non seulement avoir un impact sur leur manière de structurer leurs situations d'apprentissage, mais aussi sur la manière dont les élèves perçoivent l'espace dont ils disposent afin de faire appel à leur pensée critique selon les cours.

\section{Notes}

1 Si nous empruntions un vocable s'inscrivant plus directement dans la foulée des réflexions menées autour du concept de compétence, nous pourrions très bien traduire le terme "pratique» par celui de «savoir-agir». Nous proposons d'ailleurs un essai de définition de la pensée critique comme compétence dans l'étude doctorale de Gagnon (2008b).

2 Apportons quelques précisions sur ce dernier aspect, lequel a été développé à l'intérieur de l'article de Gagnon et Sasseville (2009). En fait, bon nombre de modèles de pensée critique de même que de taxonomies du domaine cognitif (pensons à la taxonomie de Bloom) sont structurés selon une logique allant d'habiletés dites «simples» à des habiletés considérées "complexes». Cette logique présuppose que le degré de complexité associé aux habiletés intellectuelles ne dépend pas à proprement parler du contexte, mais réside en quelque sorte «dans» l'habileté elle-même... Cependant, sans pour autant nier que certaines habiletés peuvent nécessiter le recours à d'autres habiletés pour se mettre en œuvre, nous croyons que chacune est plus ou moins complexe selon le contexte dans lequel elle prend forme. Ainsi en est-il, par exemple, de l'habileté à définir qui sera plus ou moins complexe selon le concept que nous cherchons à définir. De fait, définir une table ne semble pas relever du même niveau de complexité qu'une démarche visant à définir des concepts tels que la justice ou la vérité.

3 Par problème complexe, nous entendons une situation dans laquelle les élèves sont appelés à travailler avec des informations incomplètes, plus ou moins pertinentes, parfois contradictoires; une situation dans laquelle ils doivent chercher de l'information à partir de différentes sources et selon plusieurs aspects, dans laquelle ils doivent procéder à l'évaluation de la crédibilité et de la pertinence des informations, où il y plusieurs solutions possibles, des solutions qui sont elles-mêmes provisoires et contextuelles, et finalement, une situation dans laquelle il y a plusieurs façons d'arriver à une réponse acceptable et dans laquelle ce sont les élèves eux-mêmes qui doivent déterminer leurs critères de choix.

4 Le choix de l'APP est fondé sur les nombreuses relations présentées entre cette stratégie et la mobilisation de la pensée critique des apprenants (Gagnon, 2008b). En outre, soulignons que notre conception de l'APP se fonde sur un modèle structuré non pas sur une logique «étapiste» des processus de résolution, mais bien selon une perspective itérative. C'est pourquoi nous ne parlons pas d'étapes de résolution, mais plutôt d'espaces. Sans nier qu'il y ait, à l'intérieur de ces divers processus, des "phases» davantage dirigées vers un aspect plutôt qu'un autre, nos analyses tendent à montrer que celles-ci sont présentes, sous une forme ou sous une autre, lors de l'ensemble du processus. Les principaux espaces de résolution autour desquels sont structurés les différentes situations d'APP proposées aux élèves sont 1) l'espace problème, dans lequel les élèves explorent, déterminent et définissent le problème à résoudre; 2) l'espace solution, dans lequel les élèves planifient et mettent en œuvre leur recherche (notamment d'informations) et analysent les données recueillies; 3 ) l'espace réflexivité, dans lequel les élèves proposent des synthèses de leurs démarches par des processus d'objectivation et d'évaluation. Seulement, dans les faits, il n'est pas rare de voir des élèves qui, plongés dans un processus de recherche d'informations, prennent soin, à la lumière des textes consultés, de redéfinir le problème à résoudre, tout comme il arrive parfois que par cette redéfinition, les élèves posent un regard évaluatif sur ce qu'ils font ou 
ont fait. C'est pourquoi nous préférons parler d'espaces de résolution plutôt que d'étapes à proprement parler, et ce, afin de nous dégager d'une logique linéaire et cloisonnée des différents moments de l'APP.

5 Pour chaque sous-groupe, deux activités ont été menées en sciences, l'une touchant au tableau périodique des éléments et l'autre le concept de mole. Lors de la première activité, les élèves étaient invités à reconstituer un tableau périodique sur la base de différentes caractéristiques associées à une série d'éléments fictifs. Par la suite, ils étaient invités à réfléchir de manière épistémologique aux processus d'élaboration des modèles scientifiques. Lors de la seconde activité, les élèves étaient invités, suite à une présentation d'un assistant de recherche formé en didactique des sciences, à réfléchir de manière analogique au concept de mole en ayant recours à des enveloppes, des cure-pipes ainsi que des billes. L'un des objectifs étant de les conduire à réfléchir à la notion d'unité (de mesure) et ainsi de leur permettre de développer une meilleure compréhension du concept de mole. En histoire, la première activité touchait le concept de patrimoine, alors que la seconde portait sur la situation du Québec lors de la deuxième Guerre mondiale. Lors de l'activité sur le patrimoine, les élèves étaient d'abord invités à engager une discussion autour du concept de patrimoine puis, à l'aide d'une documentation présentant notamment l'évolution du concept de même que les principales catégories s'y rapportant, ils étaient invités à déterminer si, selon eux, les éléments contenus à l'intérieur d'une liste de 30 items devraient ou non être considérés comme faisant partie du patrimoine québécois. Cette activité se terminait avec une plénière dans laquelle chacun des sous-groupes partageait et discutait $\mathrm{du}$ fruit de leur classification ainsi que de leur conception de l'importance de protéger le patrimoine. En éthique, une recherche interdisciplinaire (îlot de rationalité; Fourez, 1997) sur la légalisation du suicide assisté a été mise en route. À cet égard, les élèves ont d'abord été engagés dans une discussion à caractère philosophique sur la question du suicide assisté. Par la suite, ils ont été invités à identifier des «boites noires» qu'ils souhaitaient ouvrir et documenter afin de structurer un argumentaire en vue de déposer une proposition au ministère de la Justice du Canada eu égard à la légalisation et/ou à la décriminalisation du suicide assisté (celui-ci étant illégal au Canada). Partant, les élèves ont été divisés en sous-groupes chargés de trouver de l'information sur chacun des volets identifiés (p. ex. éthique, social, économique, politique, légal, médical, religieux...), de l'information qui a été partagée en plénière. Lors de cet échange, les élèves étaient appelés à déterminer quelle information devrait être retenue afin de soutenir leur point de vue et de servir de point d'ancrage à leur argumentaire. Une présentation détaillée de ces activités est disponible dans le texte de Gagnon (2008b).

6 Voici une description de ces différentes interventions constitutives des pratiques critiques (PC) ainsi que des directions qu'elles peuvent prendre. Cette description est directement tirée de Gagnon (2011b). 1) Non-justifiée. L'intervention non-justifiée correspond à l'expression d'une opinion, qui est en quelque sorte le contraire d'une PC. Ce type d'intervention permet de contrebalancer des analyses qui ne seraient qu'exclusivement orientées vers l'identification de la manifestation d'une PC. Nous reprenons ici, en quelque sorte, la stratégie de Newman. 2) Justifiée. Intervention qui tend à fonder une opinion ou une action, que ce soit par une raison, un exemple, une conséquence, etc. L'intervention justifiée est un premier pas vers la mobilisation d'un critère, puisque ce dernier correspond, notamment, à une raison ayant été évaluée (Lipman, 2003). 3) Critériée. Intervention qui manifeste le recours à un critère (i.e. une raison particulièrement déterminante et objectivée): composante nécessaire (mais non suffisante) de la PC identifiée entre autres par Lipman (2003) et Bailin (2002). 4) Éthique. Toute intervention qui prend en compte les dimensions éthiques d'une question (bien, bon, mauvais, dignité...). Elle touche à la pensée responsable (sans que cette dernière s'y réduise) (Daniel, 2005) et contribue à déterminer ce qu'il y a lieu de faire (Ennis, 1985). 5) Contextuelle. En référence à Lipman 
(2003), il s'agit d'une intervention qui manifeste un souci pour mettre en évidence les circonstances ou divers cadres de référence, qu'ils soient d'ordre historique, social, disciplinaire ou paradigmatique, idéologique, familial... Cette intervention est considérée comme permettant de nuancer les jugements en évitant les généralisations hâtives. 6) Évaluative. Intervention qui se rapporte au mode propre de la PC et qui se situe dans l'axe des mégacritères identifiés par Lipman (2003): justesse, force, pertinence, cohérence, viabilité... Elle tend à «donner de la valeur», qu'elle soit d'ordre épistémologique, éthique, logique... 7) Épistémique. Intervention portant sur la valeur de vérité des savoirs, la crédibilité des sources et le rapport aux informations ; elle convoque des conceptions épistémologiques associées aux pratiques critiques. Elle touche le volet «ce qu'il y a lieu de croire» puisé chez Ennis. 8) Métacognitive. Intervention qui porte sur ses propres actions, démarches ou processus de pensée, de même que sur les outils de cognition. Elle rejoint l'axe de la pensée réflexive présent dans notre définition. 9) Autocritique et autocorrectrice. L'autocorrection est une composante de la PC (Lipman, 2003; Paul, 1990) qui présuppose une forme d'autocritique. Ainsi, elle fait de la PC une pensée autorégulatrice, consciente d'elle-même, des outils dont elle dispose et des façons adéquates de les utiliser. L'intervention autocritique porte un regard évaluatif sur ses propres pratiques, en tant qu'individu ou groupe, et, en ce sens, elle peut conduire (sans y conduire nécessairement) à l'autocorrection, laquelle manifeste un changement de vision ou de pratique (Daniel, 2005). Par ailleurs, ces interventions peuvent, selon les cas, être dirigées vers des "cibles» ou des «objets» différents. Ainsi, nous avons relevé que chacune d'entre elles pouvait porter soit sur un sujet ou un concept, sur ses propres pratiques comme individu ou groupe, sur les propos des pairs, sur ceux des experts, sur l'information consulté par le bais de différentes sources ainsi que sur le matériel utilisé par les élèves lors de l'activité (p. ex. calligraphie d'un texte, facilité d'utilisation d'un instrument...). Il s'agit, en quelque sorte, d'une déclinaison qui permet d'examiner la direction et l'usage de chacune des interventions. Voici un exemple de définition associée à chacune de ces déclinaisons:

Justifiée-soi: Intervention justifiée par une simple raison.

Justifiée-pairs: Intervention justifiée qui porte sur les propos avancés par un autre.

Justifiée-experts: Intervention justifiée à propos d'un expert ou d'une expertise.

Justifiée-information: Intervention justifiée à propos de l'information.

Justifiée-matériel: Intervention justifiée à propos du matériel.

7 Trois types d'intervention, pour lesquelles aucune occurrence n'a été relevée, ont été identifiés suivant un principe d'extension logique, à savoir 1) éthique-matériel; 2) autocritique-expert; 3) autocorrection-matériel.

8 Pour chacun des types d'intervention, les données fournies représentent le pourcentage des occurrences en fonction de toutes les interventions codées pour la même activité. Il est à noter que le total des pourcentages ne représente pas 100\% puisqu'une même intervention peut se rapporter à plus d'un type.

9 Le lecteur remarquera qu'à l'intérieur des tableaux 2, 3 et 4, l'activité sur la mole n'est pas présente. Cela participe du fait que cette activité ne comprenait pas à proprement parler d'espaces de résolution comparable, ce qui, du coup, rend difficile la mise en route d'une analyse comparative fondée sur ces espaces de résolution.

10 À l'intérieur de ces tableaux, les données associées à chacun des types correspondent au pourcentage des occurrences par rapport au total des interventions codées à l'intérieur du même espace. Ensuite, les données présentées entre parenthèses correspondent au pourcentage des occurrences pour un type d'intervention par rapport au total des interventions codées à l'intérieur de l'ensemble de l'activité. Finalement, les données présentées entre crochets correspondent au pourcentage des occurrences pour le type d'intervention traité en fonction de l'ensemble des occurrences du même type d'intervention relevé à l'intérieur de l'activité. 


\section{Rérérences}

Bailin, S. (2002). Critical thinking and science education. Science \& Education, 11, 361-375.

Barell, J. (1991). Teaching for thoughtfulness. New York: Montclair State College.

Billing, D. (2007). Teaching for transfer of core/key skills in higher education: Cognitive skills. Higher Education, 53, 483-516.

Brell, C. (1990). Critical thinking as transfer: The reconstructive integration of otherwise discrete interpretations of experience. Educational Theory, 40, (1), 53-68.

Brookfield, S. (1987). Developing critical thinkers challenging adults to explore alternative ways of thinking and acting. San Francisco: Jossey-Bass Publishers.

Brown, J. S., Collins, A. \& Duguid, P. (1989). Situated cognition and the culture of learning. Educational Researcher, 18 (1), 32-42. Educational Technology Publications. Retrieved 29. 10. 2012, from http://links.jstor.org/sici?sici=0013-189X(198901/02) 18:1<32:SCATCO $>2.0 . \mathrm{CO} ; 2-2 \&$ origin $=$ crossref

Daniel, M. F. (2005). Pour l'apprentissage d'une pensée critique au primaire. Montréal: Presses de l'Université du Québec.

Daniel, M. F. \& Gagnon, M. (2011). «Developmental process of dialogical critical thinking in pupils aged 4 to 12 years». Creative education, 2, (5), 418-428.

De Ketele, J. M. \& Roegiers, X. (1993). Méthodologie de recueil d'informations: Fondements des méthodes d'observation, de questionnaires, d'interviews et d'étude de documents. Bruxelles: De Boeck.

De Koninck, T. (2007). La crise de l'éducation. Les grandes conférence. Québec: Fides.

Delors, J. (1996). L'éducation, un trésor caché dedans. Rapport à l'UNESCO de la commission internationale sur l'éducation au vingt et unième siècle. Paris: Odile Jacob.

Dewey, J. (1933). How we think. A restatement of the relation of reflective thinking to the educative process. Boston: D.C. Heath.

Ennis, R. (1987). A taxonomy of critical thinking skills. In J. B. Baron \& R. I. Sternberg (Ed.), Teaching thinking skills: Theory and Practice (pp. 9- 26). New York: W.H. Freeman.

Ennis, R. (1989). Critical thinking and subject specificity: Clarification and needed research. Educational Researcher, 18, (3), 4-10.

Ennis, R. (1993). Critical thinking assessment. Theory into practice, 32, (3), 179-186.

Facione, S. \& Facione, P. (1996). Externalizing the critical thinking in knowledge development and critical thinking. Nursing Outlook, 44, (3), 129-136.

Fourez, G. (1997). Qu'entend-on par "îlot de rationalité» et par "îlot interdisciplinaire de rationalité»? Aster, 25, 217-225.

Fourez, G. (2005). Controverses autour de diverses conceptualisations (modélisations) des compétences transversales. Revue canadienne de l'enseignement des sciences, des mathématiques et des technologies, 5, (3), 401-412.

Gagnon, M. (2008a). La question des compétences transversales en éducation: De la métaphore du transfert à celle de la mobilisation. Revue Éducation et Formation, e-2008, 25-35.

Gagnon, M. (2008b). Étude sur la transversalité de la pensée critique comme compétence en éducation: Entre "science et technologie», histoire et philosophie au secondaire. Thèse de doctorat, Université Laval, Québec.

Gagnon, M. (2009). Conceptions d'enseignants et d'élèves du secondaire québécois sur la nature, le rôle et la place de la pensée critique dans les cours de sciences, d'histoire et de philosophie. Actes du colloque de l'Association Francophone Internationale de Recherche en Science de l'Éducation (pp. 416-430). Paris: AFIRSE.

Gagnon, M. (2010). Regards sur les pratiques critiques manifestées par des élèves de quatrième année du secondaire dans le cadre de deux activités d'apprentissage par problèmes menées en classe d'histoire au Québec. In M. A. Éthier \& J.-F. Cardin (Éd.), Histoire, musées et éducation à la citoyenneté: recherches récentes (pp. 159-181). Québec: Multimondes. 
Gagnon, M. (2011a). Examen des possibles relations entre les rapports aux savoirs et la construction d'une pensée critique chez les adolescents. Revue canadienne d'éducation, 34 , (1), 128-178.

Gagnon, M. (2011b). Proposition d'une grille d'analyse des pratiques critiques d'élèves en situation de résolution de problèmes dits complexes. Revue Recherches Qualitatives, 30, (2), 122-147.

Gagnon, M. (2011c). Penser la question des rapports aux savoirs en éducation: Clarification et besoin de recherches conceptuelles. Les Ateliers de l'Éthique / The Ethics Forum (CREUM), 6, (1), 30-42.

Gagnon, M. (2011d). Regards sur les pratiques critiques manifestées par des élèves de quatrième année du secondaire dans le cadre d'un îlot interdisciplinaire de rationalité mené en classe d'éthique au Québec. McGill Journal of Education / Revue des Sciences de l'Éducation de McGill, 45, (3), 463-494.

Gagnon, M. \& Sasseville, M. (2009). Présentation d'éléments observables dans une communauté de recherche philosophique en action: De la classification à l'organisation; De la complexité interne à la complexité contextuelle; De la linéarité à l'itérativité. In E. Marsal, T. Dobashi \& B. Weber (Ed.), Children philosophize worldwide. Theoretical and Practical Concepts (pp. 397-408). Berlin: Peter Lang.

Halpern, D. (1989). Thought and knowledge. An introduction to critical thinking. New Jersey: Lawrence Erlbaum.

Jonas, H. (1999). Le principe responsabilité: Une éthique pour la civilisation technologique. Paris: Flammarion.

Jonnaert, P. (2002). Compétences et socioconstructivisme. Un cadre théorique. Bruxelles: De Boeck.

Kurfiss, J. G. (1988). Teaching critical thinking in the disciplines. Washington, DC: Association for the study of higher education.

Lafortune, L., Prud'homme, L., Sorin, N., Aitken, A., Archambault, J., Barma, S., Beaumier, F., Boukhssimi, D., Carpentier, A., Charland, P., Durand, M.-J., Flanagan, T. D., Guillemette, S., Lebrun, M., Levebre, S., Morris, R., Myre, J., Samson, B.-G., Savoie-Zajc, L., Therriault, G., Viola, S. \& Waddington, D. (2011). Manifeste pour une école compétente. Presses de l'Université du Québec.

Landis, M., Swain, K., Friehe, M. \& Coufal, K. (2007). Evaluating critical thinking in class and online: Comparison of the Newman Method and the Facione Rubric. Communication Disorders Quarterly, 28, 135-143.

L'Écuyer, R. (1990). Méthodologie de l'analyse développementale de contenu. Sillery: PUQ.

Lessard-Hébert, M., Goyette, G. \& Boutin, G. (1990). Recherche qualitative: Fondements et pratiques. Montréal: Éditions Agence d'Arc.

Lipman, M. (2003). Thinking in education. New York: Cambridge University Press.

Maren, J.-M. van der. (2003). La recherche appliquée en pédagogie. Bruxelles: DeBoeck.

McPeck, J. E. (1981). Critical thinking and education. New York: St. Martin's.

McPeck, J. E. (1990). Critical thinking and subject specificity: A remply to Ennis. Educational Researcher, 19, (4), 10-12.

MELS. (2006). Programme de formation de l'école québécoise. Éducation préscolaire et enseignement primaire. Bibliothèque nationale du Québec.

Morin, E. (1986). La connaissance de la connaissance. Paris: Seuil.

Norris, P. (Éd.). (1992). The generalizability of critical thinking: Multiple perspectives on an educational ideal. New York: Teachers College Press.

Norris, S. \& Ennis, R. (1989). Evaluating critical thinking. CA: Midwest Publications Critical Thinking.

Paul, R. (1990). Critical thinking: What every person needs to survive in a rapidly changing world. American Educator. Retrieved October 4, 2010, from http://eric.ed.gov/ERICWebPortal/recordDetail'accno $=$ ED338557 
Perkins, D. N. \& Salomon, G. (1989). Are cognitive skills context-bound? Educational Researcher, 18, (1), 16-25.

Perrenoud, P. (2002). D'une métaphore à l'autre: Transférer ou mobiliser ses connaissances? In J. Dolz \& E. Ollagnier (Éd.), L'énigme de la compétence en éducation. Bruxelles: De Boeck.

Rey, B. (1998). Les compétences transversales en question. Collection Pédagogies (2e éd.). Paris: ESF.

Rey, B. (2011). Socle commun, e'valuation par compétences. Dijon. Consulté le 29. 10. 2012 dans http://histoire-geographie.ac-dijon.fr/spiphistoire

Siegel, H. (1985). McPeck informal logic and the nature of critical thinking. Philosophy of education, 41, 61-72.

Siegel, H. (1992). The generalizability of critical thinking skills, dispositions, and epistemology. In P. Norris (Ed.), The generalizability of critical thinking: Multiple perspectives on an educational ideal (pp. 97-108). New York: Teachers College Press.

Smith, G. (2002). Thinking skills: The question of generality. Journal of Curriculum Studies, 34, (6), 659-678.

Stewart, C. J. \& Cash, W. B. (1982). Interviewing-principles and practices. Iowa: W. C. Brown.

UNESCO. (2008). Une approche de l'éducation pour tous fondée sur les droits de l'homme. Fonds des Nations Unies pour l'enfance. Retrieved 29. 10. 2012, from http://unesdoc. unesco.org/images/0015/001588/158891f.pdf

Mots-clés: Pensée critique; transversalité; enseignement au secondaire; histoire, sciences et éthique

\section{Studie zur Transversalität von Praktiken des kritischen Denkens bei Schülerinnen und Schülern in Québéc im Rahmen von Kursen in Naturwissenschaft, Geschichte und Ethik}

\section{Zusammenfassung:}

Dieser Beitrag stellt die Ergebnisse einer explorativen Studie zur Transversalität von Praktiken kritischen Denkens bei Schülerinnen und Schülern der Sekundarstufe vor. Die explorative Studie analysiert einerseits problembasierte Lernaktivitäten in Kursen der Naturwissenschaften, Geschichte und Ethik, und wird andererseits durch einen Einblick in Interviews mit Schülerinnen und Schülern sowie Lehrpersonen ergänzt. Der Autor zeigt u.a. auf, dass die Praktiken kritischen Denkens situierte Praktiken sind, und dass deren Transversalität in einem komplexen und dynamischen Prozess verankert ist. Dieser Prozess kann anhand der Aspekte Fachgebiet, disziplinäre Lösungsräume, Problemtyp sowie Bezug zum Wissen untersucht werden.

Schlagworte: Kritisches Denken, Transversalität, Sekundarstufe, Geschichte, Naturwissenschaft, Ethik 


\section{Studio sulla trasversalità delle pratiche critiche degli adolescenti del Québec in un corso di scienze, storia ed etica}

\section{Riassunto}

Questo articolo presenta i risultati di uno studio esplorativo sulla trasversalità delle pratiche critiche degli studenti di scuola media superiore. Questo studio si appoggia, da un lato, sull'analisi delle attività di apprendimento per problemi condotte durante le lezioni di scienze, storia ed etica a partire da una griglia strutturata sugli interventi costitutivi delle pratiche critiche. Dall'altro lato, l'articolo si basa sulle interviste condotte con allievi e insegnanti. Tra gli altri risultati, l'autore indica che le pratiche critiche sono pratiche situate e che la loro trasversalità si iscrive in un processo complesso e dinamico. Che può essere descritto su quattro assi: la conoscenza disciplinare, gli spazi di soluzione, le tipologie di problema e il rapporto con il sapere.

Parole chiave: Pensiero critico, trasversalità, scuola secondaria, storia, scienze, etica

\section{Study on critical practices transversality manifested by quebecers high school students in context of problem-based learning in their science, history and ethic courses}

\section{Summary}

This article discusses the transversality of critical practices manifested by high school students in problem-based learning activities leads in their classrooms of ethic, history and science. To achieve this, the author proposes a definition of critical thinking centred on the idea of practice, and a grid based on constituent intervention types of critical practices. The results, also based on semi-structured interviews, show that the critical practices are situated and their transversality should be studied as a complex and dynamic process that could be examined from four areas: the discipline; the spaces of resolution; the types of problem; the relationships to knowledge.

Key words: Critical thinking; transversality; high school; history, science and ethic 
